\title{
Faut-il opérer les hernies hiatales?
}

Peu de questions ont fait récemment couler autant d'encre en pathologie digestive que la hernie de Thiatus æsophagien et ses consequences. En utilisant une technique adequate, les radiologistes dépistent cette lesion avec une frequence impressionnante, surtout chez les personnes âgées; et on peut suspecter sur le plan clinique

Editorial

399

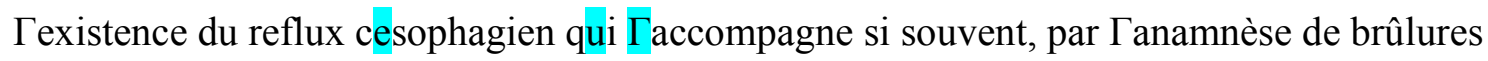
rétrosternales apparaissant au décubitus.

De là à se contenter de ce diagnostic pour expliquer un grand nombre de symptômes épigastriques, il n'y a qu'un pas. En presence d'une hernie quelconque, le réflexe thérapeutique indique la cure chirurgicale; dans le cas present, il est logique de colmater la brèche et de maintenir la grosse tubérosité gastrique dans la cavité abdomi-nale. Pour ce faire, un grand nombre de techniques ont été proposées, la multiplicité des moyens mis en ceuvre laissant déjà sous-entendre qu'aucune d'entre elles ne donne entière satisfaction.

La récente reunion extraordinaire de la Société beige de Gastro-entérologie (Acta Gastroenterologíca belgíca, vol. 21, No. special de juillet 1958) à Bruxelles, les 27 et 28 septembre 1958, avec Гactive collaboration de la Société nationale française, avait inscrit à son ordre du jour «Vavenír des hernies hiatales», envisage selon le traitement applique ou les consequences d'une abstention thérapeutique. On trouvera dans notre prochain numéro (Janvier 1959) un compte-rendu détaillé de ce Congrès. Mais nous voudrions déjà souligner quelques conclusions issues de débats particulièrement féconds.

On rend la hernie hiatale trop souvent responsable - ou seule respon-sable - des symptômes observes, parce qu'on se laisse impressionner par des cliches éloquents, comme chaque fois qu'on fournit au méde-cin des documents graphiques ou des données numériques.

Or le passage d'une partie de l'estomac dans le thorax est silen-cieux dans la majorité des cas; il s'agit d'une ectopie sans signification nosologique. Quand la hernie provoque des symptômes, ceux-ci sont divers, mais precis: palpitations et douleurs cardiaques, lorsque le volume de la malformation exerce une compression sur le cæur ou le déplace; brûlures rétrosternales en décubitus, gênant considérable-ment le sommeil; anémie par saignements «occultes» répétés. Mais la hernie hiatale est assocíée très fréquemment à d'autres affections, generatrices de manifestations qu'il est abusif d'attribuer à la hernie. En premiere ligne vient la lithiase biliaire, qu'on retrouve dans une proportion élevée de cas; la diverticulose vient en second rang, mais au niveau du duodenum notamment - elle revet une signification discutable; enfin le déplacement de l'estomac favorise Tapparition d'ulcères gastro-duodénaux, et explique certains malaises pénibles de l'angle côlique gauche, trop volontiers etiquettes «aéro-

400

Editorial

phagie». Enfin des symptôme angineux chez un porteur de hernie dia- 
phragmatique peuvent ne pas être dus à cette affection, mais à la coexistence d'une coronarite réelle, si courante après la cinquantaine. Par consequent, en presence d'une hernie hiatale, et avant toute discussion thérapeutique, un examen approfondi cardio-digestif s'impose, pour attribuer à chaque organe la part qui lui revient dans les plaintes du malade.

On conçoit fort bien en effet qu'une intervention chirurgicale, si habilement conduite et si efficace localement soit-elle, ne soulagera pas le malade ... s'il souffre d'autre chose! Avant d'envisager une intervention, les investigations nécessaires seront effectuées minu-tieusement afin d'assurer le malade qu'après Topération sur le dia-phragme, il ne continuera pas à souffrir d'un ulcère ou d'une lithiase.

Le traitement chirurgical ne doit done être discuté qu'en presence de symptômes propres - et sérieux - de la hernie hiatale, et jamais d'emblée après la simple constatation radiologique d'une lesion si souvent muette. Bien des échecs s'expliquent par une indication erronée.

II y a plus: la cure anatomique correcte de la hernie - la reposition du viscère dans $\Gamma$ abdomen est ínsuffisante si elle autoríse encore un reflux, inconvenient local majeur de la hernie hiatale; il ne s'agit done pas pour le chirurgien de restaurer seulement une situation mécanique perturbée, mais bien d'assurer la guérison des consequences de cette malformation! Pour cela, la reconstitution d'une soupape cardiale convenable s'impose, qu'elle soit réalisée par un procédé ou par un autre; et, tout en exposant longuement la technique à laquelle il donne la preference et en disant pourquoi - le chirurgien Deloyers eut Thonnêteté de reconnaître que la qualité des résultats tardifs dépen-dait plus de Гexpérience de Гopérateur que des manoeuvres répara-trices, d'ailleurs difficilement comparables entre elles.

Le succès du traitement chirurgical depend également de la forme anatomique de la lesion: en presence d'un brachy-æsophage, on aura moins de chance de succès qu'avec une hernie par glissement, dont $\Gamma$ æsophage suffisamment long permettra la reposition plus facile de $\Gamma$ organe hernie.

De plus, le malade est souvent âgé; la hernie s'est produite, au niveau du diaphragme comme ailleurs, par suite de Tatrophie ou de la senescence musculaires; ce n'est guère un terrain favorable pour la chirurgie réparatrice. 